\title{
Estado poblacional, selección de refugios y ecología espacial de las poblaciones de nóctulo grande (Nyctalus lasiopterus) y nóctulo mediano (Nyctalus noctula) en Cataluña
}

\author{
JORDI CAMPRODON ${ }^{1,2}$; DAVID GUIXÉ ${ }^{1}$
}

\author{
${ }^{1}$ Àrea de Biodiversitat. Centre Tecnològic Forestal de Catalunya \\ ${ }^{2}$ Departament d'Indústries Agroalimentàries i Ciències Ambientals. Universitat de Vic (Spain) \\ *Correo electrónico del autor: jordi.camprodon@ctfc.es
}

DOI: http://dx.doi.org/10.14709/BarbJ.6.1.2013.07

English title: Population status, roost selection and spatial ecology of the Greater Noctule Bat (Nyctalus lasiopterus) and the Common Noctule (Nyctalus noctula) in Catalonia

\begin{abstract}
We present the first results of the monitoring of the first two colonies of Greater Noctule and Common Noctule found in Catalonia, in the Zona Volcànica de la Garrotxa Natural Park and the city of Lleida, respectively. Few animals were found in each locality after a cavity checking of each locality in 2010-2012. The Great Noctule occupies a small stand with high concentration of woodpecker holes in a beech forest. The Common Noctule colony has their refuges concentrated in few European plane trees with abundant natural cavities in an urban park. Foraging areas of Great Noctule were located in the agro-forestry landscape within $30 \mathrm{~km}$ at least from the refuge stand. The Common Noctule used irrigated and fluvial areas around the city. The highly localized concentration of suitable tree cavities seems to be the determining factor in the distribution of these large bats in the study area.
\end{abstract}

Keywords: Nyctalus lasiopterus, Nyctalus noctula, spatial ecology, cavities, conservation, Catalonia.

Resumen: Se presentan los primeros resultados del seguimiento de las dos primeras colonias de nóctulo grande y nóctulo mediano encontradas en Cataluña, en el Parc Natural de la Zona Volcànica de la Garrotxa y en la ciudad de Lleida, respectivamente. Las poblaciones estaban compuestas por escasos individuos, según se desprende de la prospección de refugios potenciales en cada localidad entre 2010 y 2012. El nóctulo grande ocupaba un rodal con concentración de cavidades de pícido dentro de un hayedo, mientras la colonia de nóctulo mediano se refugiaba en un rodal de plátanos con abundantes refugios naturales en un parque urbano. Las zonas de campeo del nóctulo grande se emplazaban en el paisaje agroforestal en un radio de por lo menos $30 \mathrm{~km}$ alrededor del rodal refugio. El nóctulo mediano utilizaba las zonas de regadío y fluviales que circundan el núcleo urbano. La concentración muy localizada de buenas cavidades en árbol parece ser uno de los factores más determinantes que explica la distribución de estos quirópteros de gran tamaño en el área de estudio.

Palabras claves: Nyctalus lasiopterus, Nyctalus noctula, ecología espacial, refugios, conservación, Cataluña. 


\section{INTRODUCCIÓN}

El nóctulo grande (Nyctalus lasiopterus) y el nóctulo mediano (Nyctalus noctula) son dos de los murciélagos más raros y desconocidos de la Península Ibérica. La escasez de efectivos, su localización muy puntual y la selección de refugios en árboles grandes dificultan la localización de poblaciones. El nóctulo grande es un murciélago forestal de distribución básicamente paleártica, citado desde Marruecos a Uzbekistán, con núcleos de población discontinuos y todavía mal conocidos en la península Ibérica y el suroeste europeo (Juste in Palomo et al. 2007). Está asociado principalmente a bosques de frondosas, principalmente hayedos, a pesar de que se ha encontrado en pinares maduros y criando en viejos plátanos y palmeras de parques urbanos. El nóctulo mediano es paleártico y se ha citado desde Marruecos y Argelia hasta Asia. En la Península Ibérica y el suroeste europeo todavía se considera una especie rara y mal conocida. En 2007 solo se tenía conocimiento de 3 agrupaciones en España: dos localidades de Navarra, y otra en Aranjuez (Alcalde in Palomo et al. 2007).

El estudio de los murciélagos forestales en Cataluña ha avanzado considerablemente en los últimos años. En el 2010 se localizó un grupo de nóctulos grandes (Nyctalus lasiopterus) ocupando refugios naturales en el Parc Natural de la Zona Volcànica de la Garrotxa, el mismo año se encontró un grupo de nóctulos medianos (Nyctalus noctula) en oquedades de grandes plátanos de sombra en la ciudad de Lleida. Se trata de las primeras agrupaciones de nóctulos medianos y grandes de Cataluña, pasando a ser un nuevo y valioso enclave de los pocos que hay en la Península Ibérica, de máximo interés de conservación. Los datos anteriores publicados sobre estas dos especies en Cataluña proceden de detectores de ultrasonidos y no pueden darse como seguros (Flaquer et al. 2010). Como dato paleontológico, se halló una mandíbula de nóctulo grande del final del Pleistoceno Superior en el yacimiento neandertal del Abric Romaní, en la comarca de L'Anoia (López-García 2009).

Los resultados de este artículo emanan del proyecto de conservación de quirópteros forestales (Biosilva) del Departament d'Agricultura, Ramaderia, Pesca, Alimentació i Medi Natural de la Generalitat de Catalunya y del estudio de poblaciones de quirópteros del municipio de Lleida financiado por La Paeria.

\section{Material Y MÉtodos}

En 2010 se inició la prospección de localidades, cavidades de árboles y cajas refugio aptas para nóctulo grande y nóctulo mediano en zonas potenciales para ambas especies en algunas localidades de Cataluña. Se realizó una primera prospección en junio (época de cría) para intentar localizar colonias de reproducción y una segunda en otoño (época de apareamiento), cuando es más factible localizar ejemplares por sus gritos sociales. Se buscaron en la Fageda d'en Jordà del
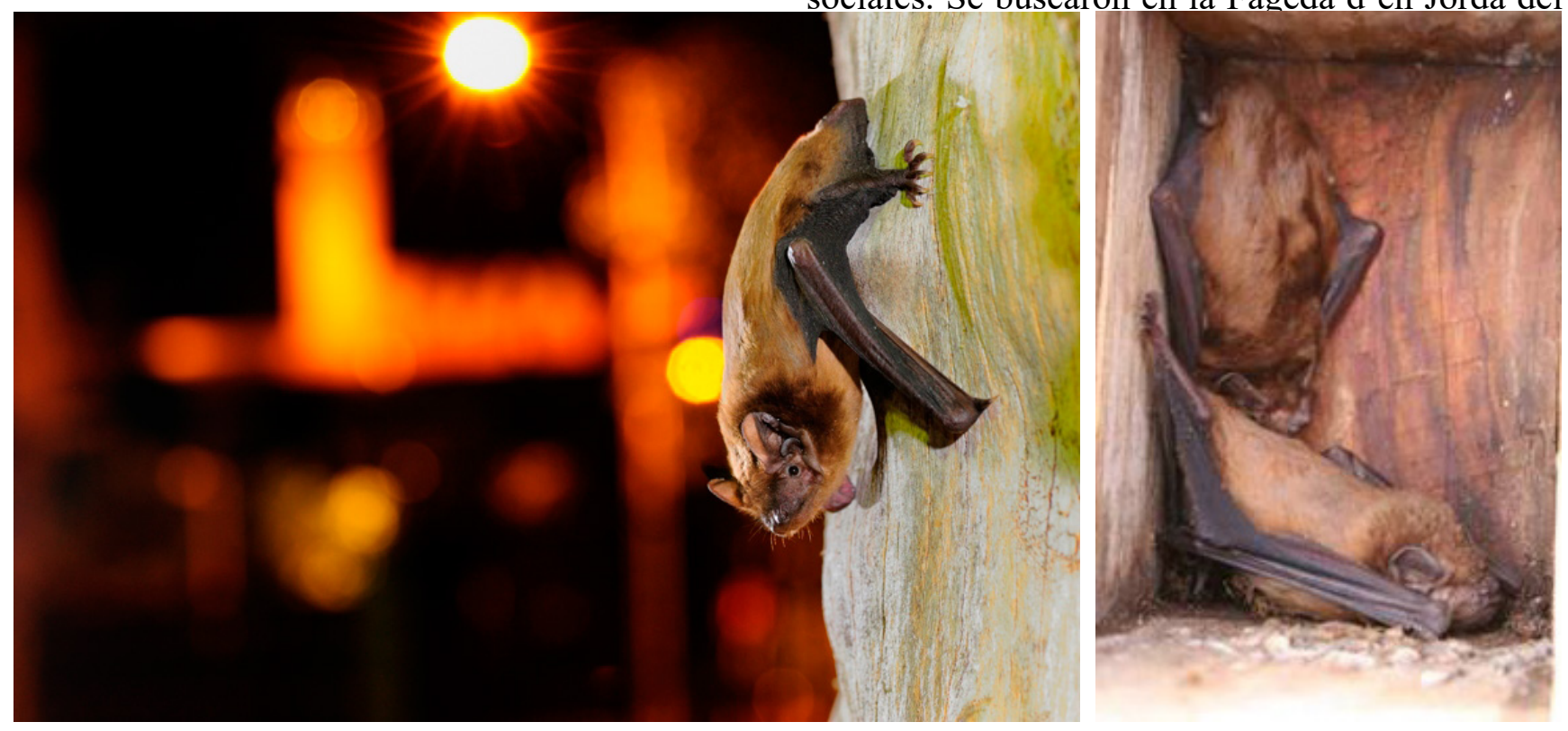

Fig 1. Machos de nóctulo grande (Nyctalus lasiopterus) en un nidal para páridos en el Parc Natural de la Zona Volcànica de la Garrotxa (izquierda) y un macho de nóctulo mediano (Nyctalus noctula) en un plátano de sombra en Lleida (derecha) Fotos: David Guixé y Jordi Bas. 
Parc Natural de la Zona Volcànica de la Garrotxa, y en los parques urbanos con grandes árboles de las ciudades de Girona, Olot y Lleida, donde se inspeccionaron las cavidades potenciales. En las distintas localidades muestreadas se emplazaron grupos de 3-4 refugios de madera (modelo Nyc06, Camprodon \& Guixé 2006) y algunas cajas Schwegler 2FN.

Para la prospección de las cavidades en árbol se utilizó un caviscopio dotado de una pequeña cámara insertada en una manguera manejable, a su vez instalada en lo alto de una pértiga telescópica que permitía llagar hasta los $9 \mathrm{~m}$ de altura. Los refugios emplazados a mayor altura se inspeccionaban trepando al árbol con escalera y cuerdas o bien subiendo a la altura de la cavidad con una grúa y revisando los agujeros aptos con un caviscopio de mango corto. Se tomaron datos dasométricos de los rodales y árboles con cavidades ocupadas.

La captura de ejemplares se efectuó mediante redes Ecotone de 9, 12 y $18 \mathrm{~m}$ o bien tapando los refugios con una bolsa-trampa. Los ejemplares capturados se marcaron con microchips AVID2323 Musicc.

La actividad de campeo y la localización de nuevos refugios se estudió mediante radioseguimiento durante los meses de octubre y noviembre de 2010-2012. Se marcaron un total de 7 nóctulos grandes y 3 nóctulos medianos con emisores Biotrack PIP3SM y Holohil BD-2C. Se siguieron en coche y a pie por la zona de refugios y de campeo. Se aprovechó un vuelo comercial en globo aerostático para averiguar si había pérdida de emisores en el área de refugios del nóctulo grande.

\section{RESUltados}

\section{Nóctulo grande (Nyctalus lasiopterus)}

\section{Estado poblacional}

Ya se tenía constancia de la presencia de la especie en el Parque Natural de la Zona Volcànica de la Garrotxa desde mayo del 2001 (Camprodon \& Guixé 2007), pero desde entonces solo conocíamos un mínimo de 3 individuos, que aparecían durante el período primavera-verano o hibernaban en una $\mathrm{u}$ otra caja del interior del hayedo. Fue en verano de 2010 cuando se halló un núcleo sedentario en cavidad natural, con un mínimo de 8 individuos. Se marcaron con microchip un total de 7 machos y
3 hembras, nueve de ellos en otoño de 2010 y 2012. Solo se recapturó una hembra. Por el momento no se han detectado indicios de reproducción. De forma paralela, en octubre de 2010 y en el transcurso de una campaña de anillamiento de aves, se capturó un individuo en el Coll de Salau (2010 m de altitud) en el Parc Natural de l'Alt Pirineu a (Bota et al. 2011).

Selección de refugios y caracterización de la estructura forestal

La población catalana localizada ocupa un hayedo monoespecífico a $650 \mathrm{~m}$ de altitud, con estructura semirregular de fustal maduro, pero con escasos árboles grandes, con diámetros medios de $23,5 \mathrm{~cm}$ y 2,2 pies/ha de $>45 \mathrm{~cm}$ de diámetro normal. Las cavidades en árbol son muy escasas excepto en un rodal que concentra la mayor parte de los refugios ocupados (unas 2,25 ha). Esté rodal se denomina el núcleo de la colonia. En este rodal se calculó una densidad de 25 refugios/ha, claramente superior del promedio de refugios estimados para el conjunto del hayedo (1,7 refugios aptos/ha). Ocupan también cavidades en árbol en zonas periféricas del mismo hayedo y algunos refugios fuera del hayedo, a veces en rodales que no destacan por la madurez del arbolado o la concentración de refugios. En las cavidades ocupadas o potenciales emplazadas fuera del núcleo de la colonia no se han encontrado otros núcleos de población, solo individuos solitarios.

Los refugios ( $n=19$ refugios) se localizaban principalmente en nidos viejos de pico picapinos y pito real en árbol vivo (16) y estaca (3) de haya (11), cerezo (2), roble (1) y álamo temblón (1), si bien también ocupaban cavidades en fisuras naturales espaciosas pero estrechas, de robles (3) y hayas (1) decrépitos (Camprodon \& Guixé 2012). También utilizaban temporalmente cajas refugio de madera para murciélago (2) (Camprodon y Guixé 2006) y nidales de párido (2) (modelo GACO 2000, Baucells et al. 2004). Una hembra invernó dos temporadas sucesivas en un viejo nidal de párido, escasamente aislado de las inclemencias meteorológicas. El recambio de refugios en los ejemplares marcados con emisor fue de 6,9 días, con un mínimo un día por cavidad ocupada hasta un máximo de 16 días. 


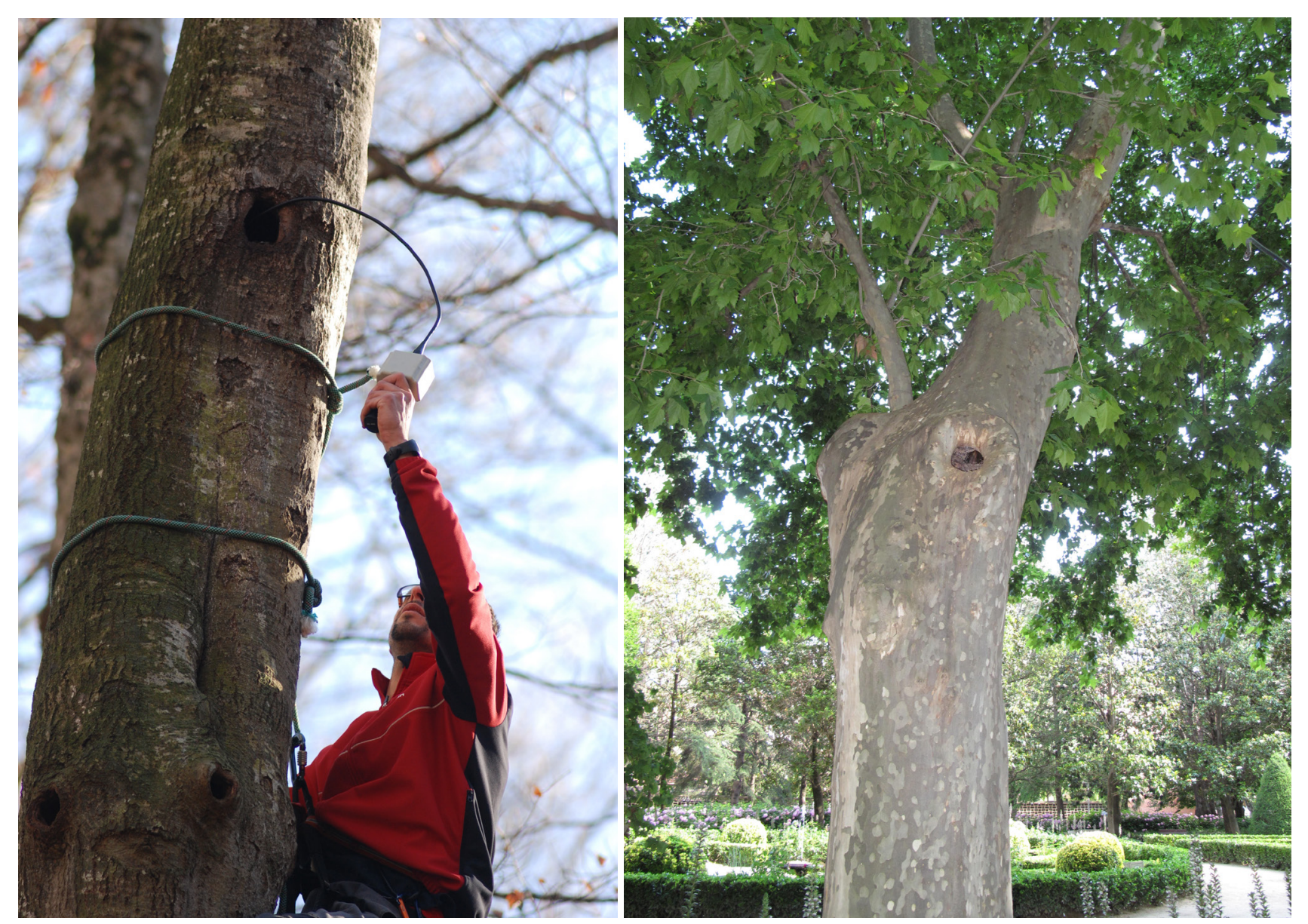

Fig. 2 Inspección con caviscopio de cavidad en estaca de haya ocupada por nóctulo grande (izquierda). Árbol con cavidad ocupada por nóctulo mediano en Lleida (derecha). Fotos: Jordi Camprodon y David Guixé.

\section{Ecología espacial}

Entre octubre y noviembre de 2010-2012 se siguieron 6 individuos, desde unos pocos días a varias semanas por animal. Una hembra marcada con emisor el 2012 desapareció la misma noche de la captura y otros individuos fueron desapareciendo de la zona en los días sucesivos, sin hallarse rastro de ellos en un radio de unos $30 \mathrm{~km}$ alrededor de la colonia. Otros dos nóctulos se arrancaron el emisor al cabo de varios días. Durante octubre concentraron la actividad de apareamiento en el núcleo de la colonia. Las áreas de campeo se emplazaron alrededor del hayedo de la colonia, en los valles circundantes de la comarca de la Garrotxa y en la llanura prelitoral de la vecina comarca del Pla de l'Estany. Cazaban a gran altura encima de un paisaje en mosaico agroforestal de bosque y cultivos de secano, dominado por frondosas (encinar, robledal, hayedo, masas mixtas) en la Garrotxa y cultivos cerealistas en el Pla de l'Estany. En octubre hasta mediados de noviembre estaban activos fuera del refugio una media de 2 h y 31 minutos (mínimo de 49 minutos y máximo de $4 \mathrm{~h}$ y 30 minutos). A medida que avanzaba el mes de noviembre reducían la actividad diaria de campeo, de forma que podían transcurrir algunos días consecutivos sin actividad fuera del refugio. En diciembre se dispersaban por el hayedo y áreas circundantes. El área perimetral (MPC) de campeo se calculó en $155,9 \mathrm{~km}^{2}$ y el área entre refugios fue de $17,9 \mathrm{~km}^{2}$. La distancia lineal máxima entre refugios utilizados por un solo individuo era de 7,9 $\mathrm{km}$ y la mediana $1,7 \mathrm{~km}$. El área de máxima de actividad de caza (Kernel 95\%) fue de $20,7 \mathrm{~km}^{2}$. La distancia máxima entre los refugios y las áreas de campeo fue de $30 \mathrm{~km}$. El análisis de un resto de excremento de un individuo capturado el 2012 contenía una pluma de ave (C. Ibáñez, com. pers.).

\section{Nóctulo mediano (Nyctalus noctula)}

\section{Estado poblacional}

En Cataluña la primera y única confirmación de un núcleo estable corresponde a la colonia localizada en un parque urbano de la ciudad de Lleida, junto al río Segre, en verano de 2010. Se descubrieron gracias a los potentes gritos sociales que emitían los machos desde las guaridas. Las capturas en cavidad o en red permitieron marcar con microchips 6 machos y 4 hembras en los veranos de 2010, 2011 y 2012, con tres recapturas 


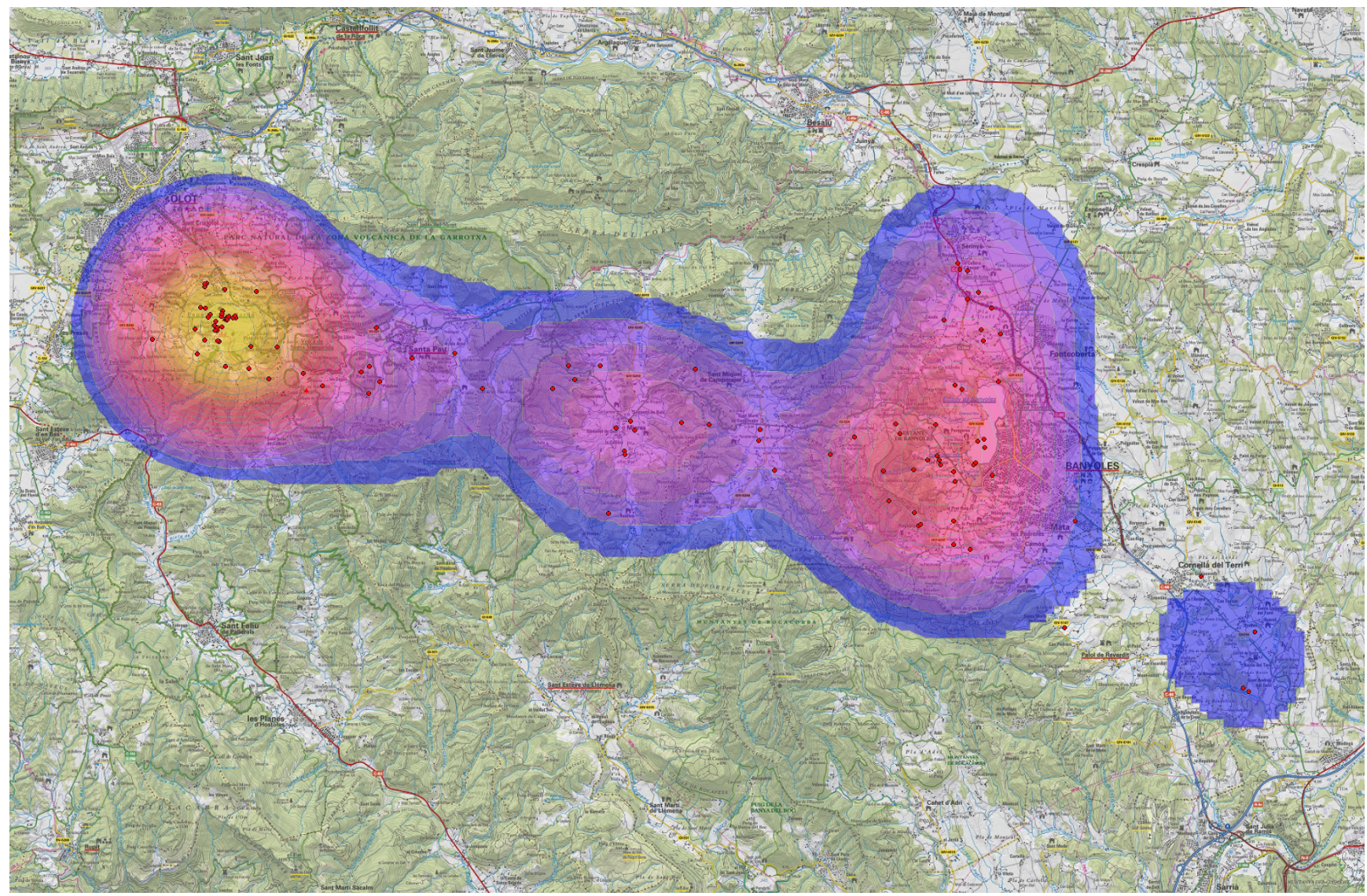

Fig. 3 Kernel 95 de 7 ejemplares de nóctulo grande en la Garrotxa-Pla de l'estany (2010-2012).

de machos. Por el momento no se han detectado indicios de reproducción.

\section{Selección de refugios y caracterización de la} estructura forestal

En verano cada macho ocupaba una cavidad en árboles diferentes muy cercanos $(<15 \mathrm{~m}) \mathrm{y}$ los machos ya estaban activos sexualmente. A mediados de septiembre llegaron las hembras (un mínimo de 3 animales). La altura media de los refugios utilizadas era de 5,8 $\mathrm{m}$, con un mínimo de 1 $\mathrm{m}$ y un máximo de $10 \mathrm{~m}(\mathrm{n}=5)$. Todas las cavidades ocupadas se hallaban en plátanos de sombra y eran de origen natural, excepto un refugio emplazado en la ventanilla de ventilación de la fachada de un edificio del centro urbano de Lleida, emplazado a 1,5 km de la colonia. Los agujeros en árbol eran pequeños de unos $4-5 \mathrm{~cm}$, con un máximo de $7 \times 10$ cm de entrada (Guixé 2010). El 2010 se instalaron cajas refugio modelo Schwegler $2 \mathrm{FN}$ que se han ocupado muy puntualmente ( 2 excrementos hallados en una caja como único indicio).

\section{Ecología espacial}

Los escasos datos de tres machos recogidos en 2011 mostraron que los nóctulos medianos utilizan los grandes plátanos de la colonia como zona residencial y campean por el área agrícola y fluvial del Segre alrededor de Lleida (comarca del Segrià). Del total de puntos de campeo ( $\mathrm{n}=$ 39 ), el $30 \%$ se localizaban en el espacio aéreo encima frutales de regadío, el $27 \%$ encima cultivos herbáceos de regadío, el 25\% encima de núcleos urbanos e industriales y el $7 \%$ por los ríos y bosques de ribera. El área vital (MPC) de dos machos de nóctulo fue de $4,54 \mathrm{~km}^{2}$ en cuatro días de radioseguimiento. El área de máxima de actividad de caza (Kernel 95\%) fue de 2,2 $\mathrm{km}^{2}$. La distancia máxima de campeo desde los refugios fue de $8 \mathrm{~km}$.

\section{DiscuSIÓN}

Durante el verano y otoño el grueso de la población localizada de nóctulo grande se refugia en un pequeño rodal de bosque, mientras que el nóctulo mediano lo hace en un grupo de árboles urbanos con refugios abundantes. Ambos forman grupos sociales de fisión-fusión, descritas para el nóctulo grande y otras especies de murciélagos forestales (Popa-Lisseanu et al. 2008). En el caso del nóctulo grande, una vez finalizado el apareamiento, 


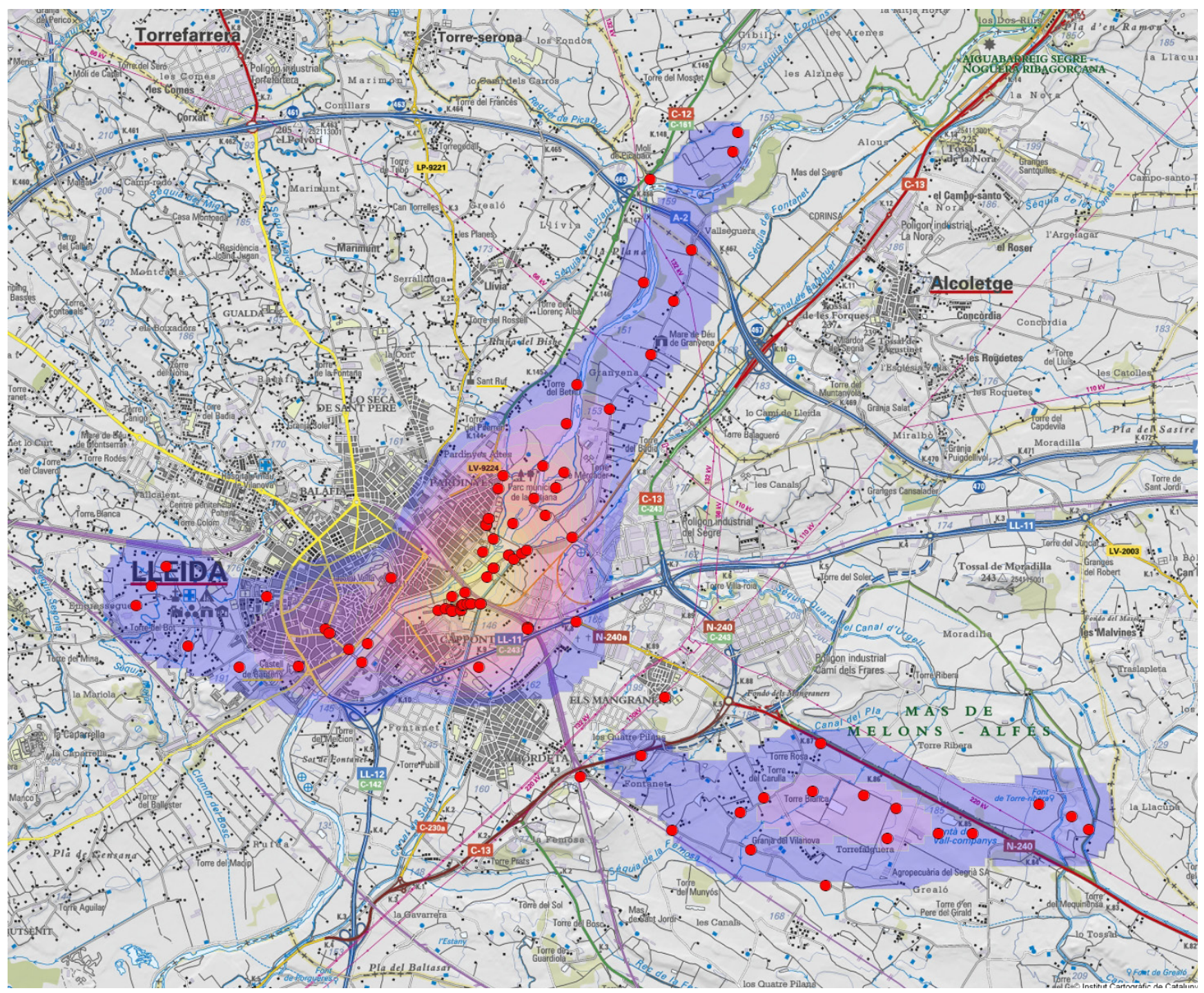

Fig. 4 Kernel 95 de 3 ejemplares de nóctulo mediano en Lleida (2011).

se dispersan por el hayedo y sus alrededores, dato coincidente con la población centroeuropea (Dietz et al. 2007). En ambas especies el tamaño poblacional de la colonia es reducido, formado por escasas decenas de individuos a lo sumo, a juzgar por las campañas de captura, prospección de refugios, escucha de gritos sociales y tasa de recaptura. No ocupan la totalidad de las cavidades disponibles de la pequeña área ocupada por el núcleo de la colonia. Parece que no hay otros núcleos de población en las inmediaciones de las colonias encontradas, a juzgar por la escasez de buenos rodales con refugios y los movimientos de los animales detectados después de campañas de prospección en verano y otoño y radioseguimiento en otoño. Sin embargo, es muy probable que existan otros núcleos de población en un área más extensa, en un radio superior a los $30 \mathrm{~km}$ en el caso del nóctulo grande, donde es posible que hayan acudido los individuos con emisor desaparecidos durante el radioseguimiento.
Las áreas de campeo del nóctulo grande probablemente coinciden con zonas migratorias de insectos y/o aves. La alimentación de paseriformes migratorios es más importante que la de insectos durante le otoño, como se ha apuntado para las poblaciones ibéricas (Ibáñez et al. 2001, PopaLisseanu et al. 2007). La tendencia de desplazarse más de $20 \mathrm{~km}$ al este de los refugios del área de estudio, hasta la llanura prelitoral, pacería indicar que en esta zona el paso migratorio en otoño es superior al interior, y compensa energéticamente los desplazamientos largos, aunque en este caso sean claramente inferiores a las distancias diarias que puede realizar la especie, por ejemplo los $40 \mathrm{~km}$ reportados para la población de Sevilla (Popa-Lisseanu et al. 2009). Los escasos datos de radioseguimiento de nóctulo mediano en Lleida solo permiten aventurar hipótesis. Se han citado distancias de caza muy superiores, por ejemplo de $26 \mathrm{~km}$ (Gebhard \& Bogdanowicz 2004). Pero en áreas con alta densidad de presas las áreas 
alimentación se concentran a menor distancia (Kronwitter 1988), efecto que podría tener el tramo léntico del Segre, bastante frecuentado por los nóctulos medianos leridanos, a pesar de su escasa superficie en comparación con el resto de cubiertas del suelo.

El nóctulo grande y el nóctulo mediano seleccionan refugios amplios, la mayoría producidos por pícido, pero también originados por fracturas en corteza y fuste (amplios y a menudo angostos), a veces comunicados por distintos agujeros de entrada. Los árboles con mayor probabilidad de presentar refugios aptos para los murciélagos son los altos, gruesos y/o añosos. Estos árboles abundan en parques urbanos, pero son raros en los bosques.

La selección del rodal de hayedo ocupado por el nóctulo grande, donde escasean los árboles grandes y añosos, se debe a la concentración nidos viejos de pícido (Guixé \& Camprodon 2010). El recambio frecuente de refugios indica que no solo es necesario disponer de refugios adecuados, sino que estas estén concentradas, formando una red de cavidades como expresan Estók et al (2007). El resto del hayedo es apto para acoger a unos pocos individuos (normalmente solitarios) fuera la época de apareamiento. La selección de refugios en el interior de rodales densos con pocas cavidades aptas, fuera del núcleo la colonia, indica un conocimiento minucioso del terreno por parte del nóctulo grande.

El nóctulo mediano sigue un comportamiento similar. El rodal de arbolado urbano que ocupa es el mejor de los posibles. A pesar que pueden ocupar temporalmente refugios en edificios, la población estudiada prefiere los refugios en árbol, por lo menos en verano y otoño, épocas que han concentrado la mayor parte de la investigación. La gestión de los árboles ornamentales de los parques urbanos de Lleida condiciona la disponibilidad de cavidades para el nóctulo mediano, debido en gran parte a la gestión de poda realizada años atrás. Esto puede condicionar que los nóctulos medianos ocupen refugios a menor altura $(5,8 \mathrm{~m}$ de media por Lleida), en comparación con la media de otras localidades europeas (por encima de los $10 \mathrm{~m}$ hasta los $19 \mathrm{~m}$ ), a pesar que el rango de alturas obtenido es concordante con datos obtenidos en otras localidades europeas (Ruczynski \& Bogdanowicz 2005). La selección de los árboles refugio también viene dada por su situación. Se ha visto que los árboles situados cerca de masas de agua o del umbral del bosque son seleccionados positivamente (Sedgeley y O'Donnell 1999, Boonman 2000), como es el caso de la colonia de Lleida, situada muy cerca del río Segre.

En conclusión, sin la existencia de rodales de árboles con concentración de buenos refugios, una y otra especie seguramente no estarían presentes en estas localidades, a pesar que existen cavidades aptas repartidas en árboles dispersos, bien por el hayedo de la Garrotxa, bien por los parques, jardines y zonas viarias de Lleida.

\section{Propuestas de conservación}

Los nóctulos ocupan varios refugios y por lo tanto varios árboles a lo largo del año, por los cuales muestran una gran fidelidad. Así pues, la corta o poda de un rodal puede conllevar la pérdida de una colonia o de agrupaciones, como se ha constado en la población de Pamplona (Alcalde, 2008). La escasez de rodales de bosque o parque con elevada densidad de refugios fuera de los localizados, hace pensar en la elevada vulnerabilidad de las poblaciones de ambas especies a escala local.

En consecuencia, se recomienda reservar rodales o pequeños grupos de árboles con refugios abundantes alrededor del árbol o árboles ocupados por nóctulos (por ejemplo, en un radio de 50 a $100 \mathrm{~m}$ ). De forma complementaria, es importante proveer para el futuro cierta densidad de árboles grandes (una densidad de 3-10 árboles/ha puede ser suficiente) en zonas cercanas a las colonias. Del mismo modo, la concentración de árboles muertos de pie de por lo menos unos $20 \mathrm{~cm}$ de diámetro normal favorecería la construcción de cavidades por los pícidos. Se puede facilitar la formación de árboles muertos anillando algunos pies rectos y gruesos.

En los espacios públicos y periurbanos es importante evitar las podas intensas de ramas con cavidades. Al mismo tiempo, es recomendable vigilar que los refugios ocupados sean accesibles a lo largo del año. Este es un aspecto importante a tener en cuenta en parques urbanos, donde las condiciones de accesibilidad pueden variar con el montaje de infraestructuras para ferias, conciertos $y$ otros acontecimientos.

El emplazamiento de cajas refugio es una medida transitoria muy buena para asentar una colonia de nóctulos, como ya proponen otros autores (Flaquer et al. 2007, Alcalde 2008, Popa- 
Lisseanu et al. 2009), en especial en rodales de bosque o en parques urbanos con escasos árboles con cavidades. Por eso, hay que asegurar una buena distribución de cajas (por ejemplo, en grupos de 2-4 cajas) colgadas de árboles cercanos a los pies ocupados (Guixé 2010), así como en zonas alternativas con árboles de buen porte, que puedan acoger a la población en caso de perturbación del rodal ocupado. Sin embargo, la mejor medida de conservación es la protección de rodales con refugios, por su naturalidad, mayores beneficios ecológicos, menor necesidad de mantenimiento y porqué los nóctulos prefieren los refugios naturales a las cajas. Finalmente resaltar la importancia de llegar a acuerdos con propietarios (públicos o privados) para preservar los árboles $\mathrm{y} / \mathrm{o}$ rodales con colonias de murciélagos.

\section{Agradecimientos}

Los resultados expuestos pertenecen al Proyecto Biosilva, conservación de quirópteros forestales del Departament d'Agricultura, Ramaderia, Pesca, Alimentació i Medi Natural de la Generalitat de Catalunya y al estudio de poblaciones de quirópteros del municipio de Lleida para La Paeria (Ayuntamiento de Lleida). Agradecemos el apoyo en todo momento de Jordi Ruiz-Olmo del Servei de Biodiversitat i Protecció dels Animals de la Generalitat de Catalunya y de Esther Fanlo de la Regidoria de Medi Ambient de La Paeria. Nos sentimos en deuda con Gerard Berengueras, Ferran Broto, Núria Fontova, David Giralt, Fermí Sort, Daniel Schönig, Aitor Domingo, Carles Durà, Xavier Moncunill, Jaume Soler-Zurita, Ádam Támas, Albert Barons, Sergi Garcia, Xavi Vergara y Rafel Rocaspana. Su ayuda fue imprescindible para el trabajo de campo. Agradecemos la colaboración del Ayuntamiento de Girona y sus técnicos, especialmente a Mercè Gómez y Ponç Feliu, a Joan Montserrat, Emili Bassols y a los técnicos del Parc Natural de la Zona Volcànica de la Garrotxa, así como al Ayuntamiento de Olot. Nuestro agradecimiento más sincero a Tomás Alcalde, Carles Flaquer y Carlos Ibáñez por sus consejos y aportación científica. Finalmente, damos las gracias a los compañeros del Área de Biodiversidad del CTFC por su apoyo en todo momento.

\section{REFERENCIAS}

Alcalde, J. T. 2007. Nyctalus noctula (Schreber, 1774) In Palomo, L. J., Gisbert, J., Blanco, J. C. (eds.). Atlas y libro rojo de los mamíferos terrestres de España. Dirección General para la Biodiversidad-SECEMSECEMU, Madrid. Pp. 228-232.

AlCALDE, J. T. 2008. El nóctulo mediano (Nyctalus noctula) en Pamplona. Colección Biodiversidad Urbana de Pamplona. Ayuntamiento de Pamplona. $52 \mathrm{p}$.

Baucells, J., Camprodon, J. Cerdeira, J. \& Vila, P. 2003. Cajas nido y comederos. Guía de las cajas nido y comederos para las aves y otros vertebrados. Lynx Edicions. Barcelona. 271 pp.

Boonman, M. 2000. Roost selection by noctules (Nyctalus noctula) and Daubenton's bats (Myotis daubentonii). J. Zool. Lond. 251: 385-389. DOI: https://dx.doi. org/10.1111/j.1469-7998.2000.tb01089.x

Bota, G., Giralt, D. \& Guixé, D. 2011. Prova pilot d'anellament en un coll de muntanya del Pirineu. L'Abellerol, Butlletí de 1'Institut Català d'Ornitologia, 43: 4-6.

Camprodon, J. \& Guixe, D. 2006. Projectes de fauna forestal. Conservació de fauna i gestió forestal. Projecte Biosilva. Memòria 2006. Centre Tecnològic Forestal de Catalunya y Departament de Medi Ambient i Habitatge de la Generalitat de Catalunya. Informe inédito.

CAmprodon, J. \& Guixé, D., 2007. Quiròpters i estructura forestal a la Fageda d'en Jordà. Collurio, Novetats de Fauna Vertebrada de la Garrotxa 1: 26.

Camprodon, J. \& Guixe, D. 2012. Projecte Biosilva: conservació de la biodiversitat i gestió forestal. Ecologia i conservació de quiròpters forestals. Centre Tecnològic Forestal de Catalunya y Departament de Medi Ambient i Habitatge de la Generalitat de Catalunya. Informe inédito.

Dietz, C. I, Helversen, O. \& Nill, D. 2007. Bats of Britain, Europe \& Northwest Africa. London: A\&C Black Publishers LtD. 
Estóк, P., Goмвкӧтö, P. \& CSERKÉsz, T. 2007. Roosting behaviour of the greater noctule Nyctalus lasiopterus Schreber, 1780 (Chiroptera, Vespertilionidae) in Hungary as revealed by radio-tracking. Mammalia 2007: 86-88.

Flaquer, C., Puig, X., Fàbregas, E., Guixé, D., Torre, I., RÀfols, R. , PÁramo, F., Camprodon, J., Cumplido, JM., Ruízjarillo, R., Baucells, A., Freixas, L. \& Arrizabalaga, A. 2010. Revisión y aportación de datos sobre quirópteros de Cataluña: propuesta de lista roja. Galemys 22 (1): 29-61.

Flaquer, C., Torre, I. \& Arrizabalaga. A. 2007. Selección de refugios, gestión forestal y conservación de los quirópteros forestales. In: Camprodon, J. \& Plana, E. (eds.). Conservación de la biodiversidad, fauna vertebrada y gestión forestal. Edicions de la Universitat de Barcelona y Centre Tecnològic Forestal de Catalunya. Barcelona. Pp. 469-488.

Gebhard J. \& Bogdanowicz W. 2004. Nyctalus noctula (Schreber, 1774) Großer Abendsegler. In: Krapp F. (ed.), Handbuch der Säugetiere Europas. 4/II. Aula Verlag, Wiebelsheim 605-694.

GuIXÉ, D. 2010. Els ratpenats de Lleida. Fase I: Inventari d'arbres dels parcs de Lleida amb cavitats d'interès utilitzats com a refugi pels ratpenats. CTFC-Ajuntament de Lleida. Informe inédito.

Guixé, D. \& CAmprodon, J., 2010. Accions per a espècies amenaçades. Projecte Biosilva: ecologia i conservació de quiròpters forestals. Centre Tecnològic Forestal de Catalunya y Departament de Medi Ambient i Habitatge de la Generalitat de Catalunya. Informe inédito.

IbÁÑEz, C., Juste, J., García-Mudarra, J. L. \& AgirRe-Mendi, P. T. 2001. Bat predation on nocturnally migrating birds. Proceedings of the National Academy of Sciences of the United States of America 58 (17): 9700 9702. DOI: https://dx.doi.org/10.1073/ pnas. 171140598
Juste, J. 2007. Nyctalus lasiopterus (Schreber, 1780) In Palomo, L. J., Gisbert, J. \& Blanco, J. C. (eds.). Atlas y libro rojo de los mamíferos terrestres de España. Dirección General para la Biodiversidad-SECEMSECEMU, Madrid. Pp 233-236.

Kronwitter, F. 1988. Population structure, habitat use and activity patterns of the noctule bat, Nyctalus noctula, revealed by radiotracking. Myotis 26:23-85.

RuCZYNSKi, I. \& BogdANOwicz, W. 2005. Roost cavity selection by Nyctalus noctula and $N$. leisleri (Vespertilionidae, Chiroptera) in Bialowieza Primeral Forest, eastern Poland. Jornal of Mammalogy 86(5): 921930. DOI: http://dx.doi.org/10.1644/15451542(2005)86[921:RCSBNN]2.0.CO;2

Popa-Lisseanu A. G., Delgado-Huertas A., Forero M. G., Rodríguez A., Arlettaz R. \& IBÉÑEZ, C. 2007. Bats' Conquest of a Formidable Foraging Niche: The Myriads of Nocturnally Migrating Songbirds. PLoS ONE 2(2): e205. doi:10.1371/journal. pone. 0000205 .

Popa-Lisseanu, A. G.; Bontadina, F., Mora, O. \& IBÁÑEZ, C. 2008. Highly structured fissionfusion societies in an aerial-hawking, carnivorous bat. Animal behaviour 75 (2): 471-482. DOI: https://dx.doi.org/10.1016/j. anbehav.2007.05.011

Popa-Lisseanu, A. G.; Bontadina, \& Ibáñez, C. 2009. Giant noctule bats face conflicting constraints between roosting and foraging in a fragmented and heterogeneous landscape. Journal of Zoology, 278: 126133. DOI: https://dx.doi.org/10.1111/ j.1469-7998.2009.00556.x

Sedgeley, J. A. \& O'Donnell, C. F. J. 1999. Roost selection by the long-tailed bat Chalinolobus tuberculatus in temperate New Zeland rainforest and its implication for conservation of bats in managed forests. Biological Conservation 88: 261-276. DOI: $\quad$ https://dx.doi.org/10.1016/S0006$\underline{3207(98) 00069-X}$ 